\title{
AS MUDANÇAS NA SAÚDE DOS PROFISSIONAIS QUE ATUAM NA ALA COVID-19
}

\author{
CHANGES IN THE HEALTH OF PROFESSIONALS WORKING IN THE \\ COVID-19 WING
}

\author{
Lidhyane Trajano de Sousa ${ }^{1}$ \\ Ankilma do Nascimento Andrade Feitosa ${ }^{2}$ \\ Talina Carla da Silva ${ }^{3}$ \\ Geane Oliveira Silva ${ }^{4}$
}

RESUMO: Este trabalho teve como objetivo identificar na literatura como a saúde dos profissionais que trabalham na ala covid-19 foi afetada. Tratando-se de um estudo de abordagem quantitativa, para a identificação de produções sobre o tema as mudanças na saúde dos profissionais que atua na ala covid-19. Adotou-se a revisão integrativa da literatura. O levantamento bibliográfico aconteceu nos meses de agosto a outubro do ano de 2021, através da BVS (Biblioteca Virtual da Saúde) e PUBMED. Foram utilizados, para busca dos artigos, os seguintes descritores e suas combinações na língua portuguesa: "Coronavírus", "Pessoal de Saúde" e "Saúde do Trabalhador". Foi realizada a pesquisa para localizar os artigos publicados entre os anos de 2019 a 2021, em seguida realizada a leitura dos resumos e dos textos na íntegra, sendo assim, incluídos os estudos que tenham citação a saúde do trabalhador do setor covid. No que diz respeito aos resultados, ao analisar a temática estudada, observou-se na literatura que a saúde dos profissionais que ofertam serviços aos pacientes acometidos por SARS COV 2 que existe uma ênfase para a questão dos EPI, medidas preventivas excessivas, acarretando alterações físicas evidentes, outras pesquisas também enfatizam a saúde psicológicas desses trabalhadores, devido a própria dinâmica do processo assistencial mediante a pandemia e sobrecarga de trabalho. Concluindo, a saúde dos profissionais de saúde foi afetada pelo fato dos mesmos possuírem uma maior chance de adquirir a patologia, sofrer pressão psicológica, assim como estarem mais susceptíveis a

\footnotetext{
1 Graduanda do curso de Enfermagem da Faculdade Santa Maria de Cajazeiras. lidyanepe@hotmail.com.

2 Enfermeiro. Doutora em Ciência da Saúde. Docente da Faculdade Santa Maria - FSM. ankimar@hotmail.com.

3 Enfermeira. Doutora em Ciências da Saúde. Docente da Faculdade Santa Maria - FSM. Talinacarla@hotmail.com.

4 Enfemeira. Mestre em enfermagem. Docente da Faculdade Santa Maria - FSM. Geane32.silva@gmail.com.
} 
complicações da doença, pois este público encontra-se mais exposto devido uma maior exposição ao vírus.

Palavras chave: Coronavírus. Pessoal de Saúde. Saúde do trabalhador.

\begin{abstract}
This study aimed to identify in the literature how the health of professionals working in the covid-19 ward was affected. This is a study with a quantitative approach, to identify productions on the subject of changes in the health of professionals working in the covid ward-19. An integrative literature review was adopted. The bibliographic survey took place from August to October of 2021, through the VHL (Virtual Health Library) and PUBMED. The following descriptors and their combinations in Portuguese were used to search for articles: "Coronavirus", "Health Personnel" and "Worker's Health". The search was carried out to locate the articles published between the years 2019 to 2021, then the abstracts and texts were read in full, thus including studies that mention worker health in the covid sector. With regard to the results, when analyzing the subject studied, it was observed in the literature that the health of professionals who offer services to patients affected by SARS COV 2 that there is an emphasis on the issue of PPE, excessive preventive measures, causing physical changes evident, other studies also emphasize the psychological health of these workers, due to the dynamics of the care process due to the pandemic and work overload. In conclusion, the health of health professionals was affected by the fact that they have a greater chance of acquiring the pathology, suffering psychological pressure, as well as being more susceptible to complications from the disease, as this public is more exposed due to greater exposure to virus.
\end{abstract}

Keywords: Coronavirus. Worker's Health. Health Personnel. 


\section{INTRODUÇÃO}

O Covid-19 trata-se de uma comorbidade que encontra-se predominante a nível mundial acometendo principalmente o sistema respiratório, onde a Organização Mundial da Saúde (OMS) tornou público sua existência em dezembro de 2019 no que diz respeito a descoberta de um novo vírus encontrado em um paciente hospitalizado com pneumonia na China em uma cidade chamada de Wuhan, onde posteriormente foi denominado de SARS-COV-2, pertencente ao subgênero Sarbecovírus da família Coronaviridae, é o sétimo coronavírus a infectar seres humanos. Meses depois da descoberta do primeiro caso tomou uma proporção tão intensa na qual tornou-se uma pandemia mundial nunca visa antes na história contemporânea (RIBEIRO et al., 2020).

O COVID-19 é de fácil disseminação espalhando-se por meio de gotículas respiratórias principalmente durante o contato entre indivíduos, sendo classificado como uma doença infecciosa aguda na qual é considerada potencialmente grave, onde é classificada em casos assintomáticos quando não a presença de sintomas, casos leves quando não ocorre sinais de gravidade, existindo apenas a presença de sintomas brandos. Há também os casos moderados onde também não apresentam sinais de gravidade podendo existir um pequeno comprometimento pulmonar e os casos graves nos quais ocorrem diminuição da saturação (< 95\%) havendo a necessidade de suporte ventilatório, dispneia, hipóxia, distúrbios de coagulação entre outros sinais de gravidade. (PRADO et al., 2020).

Em 9 de abril de 2020, o Covid-19 resultou em 1.521 .252 casos e 92.798 mortes em todo o mundo, porem um ano após esses dados de acordo com o boletim epidemiológico da Organização Mundial de Saúde a doença tomou grandes proporções por todos os países. No momento há 147.539 .302 casos confirmados e 3.116.444 de óbitos em todo o mundo. Segundo o boletim informativo da situação epidemiológica, o Brasil se encontra com 14.369 .423 casos confirmados e 391.936 
óbitos, sendo destes na Paraíba 288.282 casos confirmados e 6.676 óbitos (BURRER et al., 2020).

Os profissionais de saúde $(\mathrm{PCH})$ são trabalhadores que exercem suas funções trabalhistas em unidades de saúde podendo vir a ser remunerados ou não, sendo esses essenciais existindo potencial de exposição direta ou indireta a pacientes ou materiais infecciosos. Os trabalhadores da saúde nos quais atuam diretamente na linha de frente ao cuidado ofertados a pacientes acometidos com Covid- 19 tem sido os mais vulneráveis a adquirir a patologia devido a exposição direta ao vírus, além disso vem os traumas psicológicos em meio a tantos fatores que estão relacionados as causas do estresse por esses profissionais, podemos citar como exemplos a falta de EPls (equipamento de proteção individual), a sobrecarga de trabalho, exaustão física e mental (CHUNG, 2020).

Com o surgimento da pandemia do Covid-19 acarretou em inúmeras mudanças na rotina do profissional de saúde principalmente no ambiente hospitalar, como por exemplo, o surgimento de uma ala isolada para sintomáticos respiratórios acometidos por SARS-COV-2 e com isto medo do desconhecido por se tratar de uma doença que pouco se sabe até o momento, doença esta que surgiu de forma grotesca pegando a todos de surpresa, gerando uma caos na saúde onde o número de paciente hospitalizados aumentou de forma alarmante deixando os profissionais de saúde sobrecarregados. (WIERSINGA et al., 2020).

Espera-se que este trabalho contribua tanto de forma científica como também social. Científica, pelo fato de a escassez de artigos devido ser uma nova comorbidade que pouco se sabe até o momento, principalmente no que diz respeito à saúde dos profissionais que lidam no enfrentamento desta pandemia. Acredita-se também que, quanto à forma social, abordará uma temática que está presente nos dias atuais, assim como também vem sendo colocada em pauta cada dia mais pela grande proporção que vem tomando a nível mundial, onde existe uma carência de políticas públicas que de fato se sobressaiam minimizando os altos índices de morbimortalidade da Covid-19.

Considerando a realidade circundante, tem-se verificado grande importância em ampliar novos estudos na área, pois a invisibilidade, no que diz respeito à saúde dos profissionais na linha de frente do Covid para melhor realizar uma avaliação da 
condição de trabalho em que foram expostos e a proporção dos danos causados nesses profissionais de saúde nesse momento conturbador é de grande importância para que de certa forma venha a conhecimento de todos quais foram e ainda são as consequências causadas pelo processo de trabalho desempenhado arduamente nos centros hospitalares. Estes fatos remetem à seguinte situação-problema: Como a saúde dos profissionais que trabalham na ala covid-19 foi afetada? No intuito de responder a estes questionamentos, objetivou-se identificar na literatura como a saúde dos profissionais que trabalham na ala covid-19 foi afetada.

\section{METODOLOGIA}

Trata-se de estudo de abordagem quantitativa, para a identificação de produções sobre o tema as mudanças na saúde dos profissionais que atua na ala covid-19. Adotou-se a revisão integrativa da literatura, uma vez que ela contribui para o processo de sistematização e análise dos resultados, visando à compreensão de determinado tema, a partir de outros estudos independentes (MENDES; SILVEIRA; GALVÃO, 2008).

Ela está dividida em seis etapas: delimitação do tema ou elaboração da pergunta norteadora; busca ou amostragem na literatura; coleta de dados; analise crítica dos estudos incluídos; discursão dos resultados e apresentação da revisão integrativa e síntese do conhecimento (SOARES et al., 2014).

Sendo assim, utilizou-se seguinte questão norteadora Como a saúde dos profissionais que trabalham na ala covid-19 foi afetada? e para o alcance dos objetivos foram seguidas as seguintes etapas: identificação do tema e seleção da questão de pesquisa; estabelecimento de critérios de inclusão e exclusão; identificação dos estudos pré-selecionados e selecionados, realizando a leitura criteriosa dos títulos, resumos e palavras-chave de todas as publicações completas para determinar se estavam conforme os critérios de inclusão do estudo; categorização dos estudos selecionados; análise e interpretação dos resultados e 
finalmente apresentação da revisão/síntese do conhecimento para a elaboração do artigo (MENDES; SILVEIRA; GALVÃO, 2008).

O levantamento bibliográfico aconteceu nos meses de agosto a setembro do ano de 2021, através da BVS (Biblioteca Virtual da Saúde) e PUBMED. Foram utilizados, para busca dos artigos, os seguintes descritores e suas combinações na língua portuguesa: "Coronavirus", "Pessoal de Saúde" e "Saúde do Trabalhador".

Foi realizado a pesquisa para localizar os artigos publicados entre os anos de 2019 a 2021, em seguida foi realizada a leitura dos resumos e dos textos na íntegra, sendo assim, foram incluídos os estudos que tenham citação a saúde do trabalhador do setor covid.

Após essa leitura foi realizado a seleção dos estudos primários, de acordo com a questão norteadora e os critérios de inclusão previamente definidos. Todos os estudos foram identificados por meio da estratégia de busca onde foi inicialmente avaliar a análise dos títulos e resumos. Nos casos em que os títulos e os resumos não se foram suficientes para definir a seleção inicial, procedeu-se à leitura da íntegra da publicação. Os critérios de inclusão foram: artigos publicados na integra; publicados em português que retratarem a temática abordada e artigos publicados nos referidos bancos de dados entre os anos de 2019 a 2021. Como critérios de exclusão os artigos publicados antes de 2019; estudos que não atenderem os critérios de inclusão mencionados. Logo após, em seguida, foi realizado um levantamento bibliográfico preliminar, como um estudo exploratório, com a finalidade de proporcionar familiaridade com a área de estudo, por meio das bases de dados referidas anteriormente.

Os artigos que compõem esse estudo foram escolhidos por meio da leitura do título, resumo e do trabalho na íntegra, respectivamente. Para a coleta das informações relevantes dos artigos foi criado um instrumento que abrangeu os seguintes dados: título, autores, ano de publicação, periódico, objetivo de estudo, delineamento, público alvo estudado e local e síntese de resultados. A sistematização das informações do material obtido ocorreu mediante análise dos artigos por meio da construção de quadros-resumo. Após a leitura exaustiva desses artigos buscou-se os resultados semelhantes discutidos e confrontados em categorias. 
Esta pesquisa seguiu os preceitos da ética e bioética, mesmo não sendo submetida ao Comitê de Ética e Pesquisa.

Quadro 1 - A metodologia da escolha de artigos.

\begin{tabular}{|c|c|c|c|}
\hline Base de dados & $\begin{array}{c}\text { Artigos } \\
\text { encontrados }\end{array}$ & Filtro & $\begin{array}{c}\text { Correspondem ao } \\
\text { tema }\end{array}$ \\
\hline BVS & 3.026 & 95 & 05 \\
\hline PUBMED & 353 & 302 & 01 \\
\hline
\end{tabular}

Fonte: Elaborado pelos autores (2021).

\section{RESULTADOS}

Dentre os artigos inclusos na presente revisão integrativa, a maioria trouxe como objeto de estudo a equipe de profissionais de saúde, sendo de sua grande maioria do ano 2020, onde apenas um é do ano 2021, sendo publicados por profissionais de saúde.

No que diz respeito ao delineamento metodológico dos estudos trabalhados foi-se possível observar: pesquisas qualitativas, descritivas, exploratórias, revisões integrativas de literatura e estudos transversais.

Na tabela 1, e no quadro 2 apresenta-se a síntese dos estudos utilizados para compor a presente pesquisa. 
Tabela 1 - Apresentação da síntese de artigos incluídos na revisão integrativa.

\begin{tabular}{|c|c|c|c|}
\hline Procedência & Título do artigo & Autores Periódico (ano) & $\begin{array}{l}\text { Modalidade } \\
\text { científica }\end{array}$ \\
\hline BVS & $\begin{array}{l}\text { Prevenção relacionada à } \\
\text { exposição ocupacional do } \\
\text { profissional de saúde no } \\
\text { cenário de COVID-19 }\end{array}$ & $\begin{array}{l}\text { GALLASCH, Cristiane Helena; CUNHA, } \\
\text { Márcia Lima da; PEREIRA, Larissia } \\
\text { Admá de Souza; SILVA, João Silvestre. } \\
2020\end{array}$ & Artigo \\
\hline BVS & $\begin{array}{l}\text { Trabalhadores(as) da } \\
\text { saúde e a COVID-19: } \\
\text { condições de trabalho à } \\
\text { deriva? }\end{array}$ & $\begin{array}{l}\text { VEDOVATOA, Tatiana Giovanelli; } \\
\text { ANDRADE, Cristiane Batista; SANTOS, } \\
\text { Daniela Lacerda; BITENCOURTD, } \\
\text { Silvana Maria; ALMEIDA, Lidiane } \\
\text { Peixoto de; SAMPAIO, Jéssyca Félix da } \\
\text { Silva. } 2020\end{array}$ & Artigo \\
\hline PUBMED & $\begin{array}{l}\text { Saúde mental } \\
\text { profissionais des } \\
\text { (profissionais de saúde } \\
\text { uma revisão): } \\
\text { intervenções } \\
\text { organizacionais } \\
\text { implementadas } \\
\text { instituições locais por } \\
\text { lidar com os novos } \\
\text { desafios psicossociais } \\
\text { resultantes do COVID-19 }\end{array}$ & $\begin{array}{l}\text { BUSELLI, Rodolfo; CORSI, Martina ; } \\
\text { VELTRI, Antonello; BALDANZI, Sigrid ; } \\
\text { CHIUMIENTO, Martina. } 2021\end{array}$ & Artigo \\
\hline BVS & $\begin{array}{l}\text { A saúde mental da } \\
\text { enfermagem no } \\
\text { enfrentamento da COVID- } \\
19 \text { em um hospital } \\
\text { universitário regional }\end{array}$ & $\begin{array}{llr}\text { DAL'BOSCO, } & \text { Eduardo } & \text { Bassani; } \\
\text { FLORIANO, Lara Simone } & \text { Messias; } \\
\text { SKUPIEN, } & \text { Suellen } & \text { Vienscoski; } \\
\text { ARCARO, Guilherme; } & \text { MARTINS, } \\
\text { Alessandra } & \text { Rodrigues; } & \text { ANSELMO, } \\
\text { Aline Cristina Correa. } 2020 & \\
\end{array}$ & Artigo \\
\hline BVS & $\begin{array}{l}\text { Covid-19: Por que a } \\
\text { proteção } \\
\text { trabalhadores } \\
\text { trabalhadoras da saúde é } \\
\text { prioritária no combate à } \\
\text { pandemia? }\end{array}$ & $\begin{array}{l}\text { HELIOTERIO, Margarete Costa; } \\
\text { LOPES, Fernanda Queiroz Rego de } \\
\text { Sousa; SOUSA, Camila Carvalho de; } \\
\text { SOUZA, Fernanda de Oliveira; PINHO, } \\
\text { Paloma de Sousa; SOUSA, Flávia } \\
\text { Nogueira e Ferreira de; ARAÚJO, Tânia } \\
\text { Maria de. } 2020\end{array}$ & Artigo \\
\hline BVS & $\begin{array}{l}\text { Mapeamento dos } \\
\text { profissionais de saúde no } \\
\text { brasil: alguns } \\
\text { apontamentos em vista } \\
\text { da crise sanitária da } \\
\text { covid-19 }\end{array}$ & $\begin{array}{l}\text { LOPEZ, Felix Garcia; PALOTTI, Pedro } \\
\text { Lucas De Moura; BARBOSA, Sheila } \\
\text { Cristina Tolentino; KOGA, Natália } \\
\text { Massaco. } 2020\end{array}$ & Livro \\
\hline
\end{tabular}

Fonte: Elaborado pelos autores (2021). 
Quadro 2 - Aspectos das produções científicas identificadas na pesquisa: objetivos, delineamento metodológico utilizado, público alvo e síntese dos resultados.

\begin{tabular}{|c|c|c|c|c|}
\hline № & OBJETIVO & DELINEAMENTO & $\begin{array}{l}\text { PUBLICO } \\
\text { ALVO }\end{array}$ & SÍNTESE DOS RESULTADOS \\
\hline 1. & $\begin{array}{l}\text { Descrever } \\
\text { principais as } \\
\text { recomendações sobre } \\
\text { ações de prevenção } \\
\text { de contágio } \\
\text { relacionadas à } \\
\text { exposição ocupacional } \\
\text { dos profissionais de } \\
\text { saúde atuantes frente } \\
\text { à COVID-19, } \\
\text { disponíveis até março } \\
\text { de 2020. }\end{array}$ & $\begin{array}{l}\text { Pesquisa } \\
\text { qualitativa, } \\
\text { descritiva e } \\
\text { exploratória. }\end{array}$ & $\begin{array}{l}\text { Profissional } \\
\text { de saúde }\end{array}$ & $\begin{array}{l}\text { A atual pandemia da doença } \\
\text { causada pelo novo coronavírus } \\
\text { SARS-CoV-2 tem transmissão } \\
\text { favorecida pelo contato próximo e } \\
\text { desprotegido com secreções ou } \\
\text { excreções de pacientes infectados, } \\
\text { principalmente por meio de } \\
\text { gotículas salivares. Práticas } \\
\text { organizacionais de prevenção } \\
\text { devem ser previstas antes da } \\
\text { chegada do paciente ao serviço de } \\
\text { saúde, reduzindo o fluxo de } \\
\text { atendimento, no primeiro } \\
\text { atendimento e durante as ações } \\
\text { assistenciais, para minimizar a } \\
\text { exposição ocupacional ao agente } \\
\text { biológico. Profissionais de saúde } \\
\text { classificados como grupo de risco } \\
\text { devem ser afastados de atividades } \\
\text { de risco de contaminação. Aqueles } \\
\text { contaminados ou adoecidos devem } \\
\text { permanecer em quarentena para } \\
\text { minimizar a propagação da } \\
\text { CovID-19. C }\end{array}$ \\
\hline 2. & $\begin{array}{l}\text { Analisar as condições } \\
\text { de trabalho dos } \\
\text { profissionais de saúde } \\
\text { que atuam na } \\
\text { pandemia de COVID- } \\
19 \text {, no Brasil, com } \\
\text { base em reportagens } \\
\text { publicadas na internet } \\
\text { por veículos de } \\
\text { comunicação } \\
\text { jornalística }\end{array}$ & $\begin{array}{l}\text { Esta pesquisa } \\
\text { tem caráter } \\
\text { qualitativo }\end{array}$ & $\begin{array}{l}\text { Profissional } \\
\text { de saúde }\end{array}$ & $\begin{array}{l}\text { As reportagens evidenciaram } \\
\text { condições de trabalho inadequadas } \\
\text { por ausência e/ou precariedade } \\
\text { dos EPI; continuidade do trabalho } \\
\text { de profissionais de saúde com } \\
\text { comorbidades; adoecimento e } \\
\text { mortes pela COVID-19; tensão e } \\
\text { medo de serem infectados(as) e de } \\
\text { lidar com o adoecimento e morte } \\
\text { de colegas; dificuldades no acesso } \\
\text { aos testes de COVID-19 e para } \\
\text { afastamento do trabalho para } \\
\text { tratamento; desistências de } \\
\text { trabalhar na atividade; necessidade } \\
\text { de atualização rápida para o } \\
\text { cuidado em saúde na COVID-19. }\end{array}$ \\
\hline 3. & $\begin{array}{l}\text { O objetivo da presente } \\
\text { revisão é verificar as }\end{array}$ & $\begin{array}{l}\text { Pesquisa } \\
\text { qualitativa. }\end{array}$ & $\begin{array}{l}\text { Profissional } \\
\text { de saúde }\end{array}$ & $\begin{array}{l}\text { Evidenciamos como poucos países } \\
\text { publicaram protocolos específicos }\end{array}$ \\
\hline
\end{tabular}




\begin{tabular}{|c|c|c|c|c|}
\hline & $\begin{array}{l}\text { intervenções em todo } \\
\text { o mundo na redução } \\
\text { do estresse nos HCWs } \\
\text { durante o surto de } \\
\text { COVID-19 }\end{array}$ & & & $\begin{array}{l}\text { de intervenção de apoio } \\
\text { psicológico para os HCWs. Todos } \\
\text { os programas foram desenvolvidos } \\
\text { em hospitais associados à } \\
\text { universidade e destacaram a } \\
\text { importância da colaboração } \\
\text { multidisciplinar. Todos eles tinham } \\
\text { como objetivo gerenciar os } \\
\text { desafios psicossociais aos HCW's } \\
\text { durante a pandemia, a fim de } \\
\text { prevenir problemas de saúde } \\
\text { mental. }\end{array}$ \\
\hline 4. & 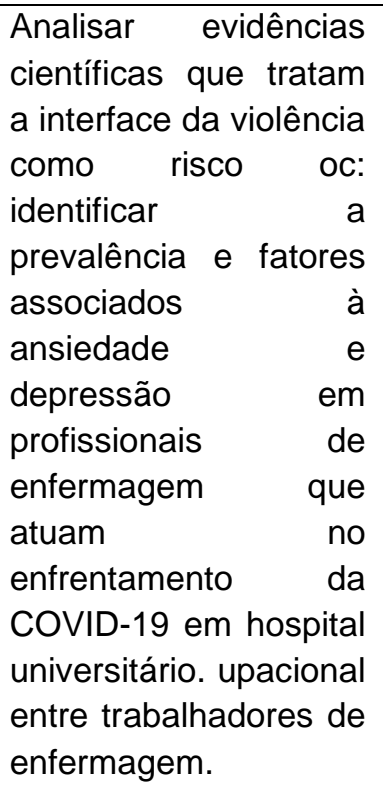 & $\begin{array}{l}\text { Estudo } \\
\text { observacional } \\
\text { transversal }\end{array}$ & $\begin{array}{l}\text { Equipe de } \\
\text { enfermage } \\
\mathrm{m} .\end{array}$ & $\begin{array}{l}\text { Houve prevalência de ansiedade } \\
(48,9 \%) \text { e de depressão }(25 \%) \text {. A } \\
\text { maioria da amostra foi composta } \\
\text { por mulheres, com mais de } 40 \\
\text { anos, casadas ou em união } \\
\text { estável, de cor branca, com ensino } \\
\text { superior ou pós-graduação, com } \\
\text { renda superior a } \$ 3.000,00 \text {, } \\
\text { concursadas, com regime de } \\
\text { trabalho de } 40 \text { horas semanais e } \\
\text { tempo de atuação no hospital de } 1 \\
\text { a } 5 \text { anos. }\end{array}$ \\
\hline 5. & $\begin{array}{l}\text { Objetiva-se sumarizar } \\
\text { e sistematizar } \\
\text { aspectos relativos às } \\
\text { condições de trabalho } \\
\text { e de saúde dos(as) } \\
\text { trabalhadores(as) da } \\
\text { saúde nessa } \\
\text { pandemia, enfatizando } \\
\text { a situação no Brasil, } \\
\text { experiências exitosas } \\
\text { na proteção do } \\
\text { trabalho em saúde em } \\
\text { outros países e } \\
\text { recomendações para o } \\
\text { contexto brasileiro. }\end{array}$ & $\begin{array}{l}\text { Pesquisa } \\
\text { qualitativa, } \\
\text { descritiva e } \\
\text { exploratória. }\end{array}$ & $\begin{array}{l}\text { Profissionai } \\
\text { s de saúde. }\end{array}$ & $\begin{array}{l}\text { Destacam-se } \\
\text { recomendações: revisão de fluxos } \\
\text { de atendimento e definição de } \\
\text { características e condições para } \\
\text { cada etapa de atendimento; } \\
\text { estabelecimento da Covid-19 como } \\
\text { doença relacionada ao trabalho } \\
\text { para os grupos expostos; registro } \\
\text { efetivo da 'ocupação' nos sistemas } \\
\text { de informação; estabelecimento de } \\
\text { condições especiais para execução } \\
\text { do trabalho na situação de } \\
\text { epidemia; atenção às jornadas } \\
\text { laborais e ações para redução de } \\
\text { estressores ocupacionais. A } \\
\text { atuação desses(as) } \\
\text { trabalhadores(as) é elemento } \\
\text { central no enfrentamento da } \\
\text { pandemia, portanto, o plano de } \\
\text { combate ao Covid-19 deve incluir } \\
\text { proteção e preservação de sua }\end{array}$ \\
\hline
\end{tabular}




\begin{tabular}{|c|c|c|c|c|}
\hline & & & & saúde física e mental. \\
\hline 6. & $\begin{array}{lr}\text { Identificar } & \text { a } \\
\text { prevalência e os } \\
\text { fatores associados à } \\
\text { violência ocupacional } \\
\text { na equipe de } \\
\text { enfermagem. }\end{array}$ & $\begin{array}{l}\text { Estudo } \\
\text { transversal. }\end{array}$ & $\begin{array}{l}\text { Profissionai } \\
\text { s de saúde. }\end{array}$ & $\begin{array}{l}\text { Como apresentado pelos dados } \\
\text { explorados nesta nota técnica, a } \\
\text { crise sanitária que se avizinha } \\
\text { lidará com um contexto de } \\
\text { assimetrias prévias nas } \\
\text { capacidades estatais dos estados } \\
\text { brasileiros. Entendendo as } \\
\text { capacidades como estoque de } \\
\text { recursos acumulados pelos entes } \\
\text { estatais, os dados retratam tal } \\
\text { assimetria no âmbito dos recursos } \\
\text { humanos passíveis de serem } \\
\text { mobilizados para o enfrentamento } \\
\text { da crise. }\end{array}$ \\
\hline
\end{tabular}

Fonte: Elaborado pelos autores (2021).

\section{DISCUSSÃO}

Ao estudar a literatura pertinente ao assusto abordado no que diz respeito a saúde dos profissionais prestadores de serviços de saúde a paciente diagnosticado por Covid é possível observar uma fragilidade com relação a medidas preventivas, assim como também a falta de EPl's ou seu uso errôneo, a vulnerabilidade na saúde mental e física desses trabalhadores.

A pandemia do covid-19 ocasionou diversas mudanças no cenário do ambiente de trabalho a nível hospitalar e na atenção básica, onde uma boa parte de profissionais de saúde foram infectados devido a aproximação constante a pacientes contaminados pelo vírus sem a devida proteção. Durante a assistência a esses pacientes, esses profissionais necessitam usar incessantemente equipamentos de proteção individual (EPI) e esses equipamentos muitas vezes sufocam e impedem de realizar procedimentos (LOPES et al., 2020).

Os trabalhadores da saúde nos quais atuam diretamente na linha de frente ao cuidado ofertados a pacientes acometidos com Covid- 19 tem sido os mais vulneráveis a adquirir a patologia devido a exposição direta ao vírus, além disso vem os traumas 8 psicológicos em meio a tantos fatores que estão relacionados as causas do estresse por esses profissionais, podemos citar como exemplos a falta de 
EPIs (equipamento de proteção individual), a sobrecarga de trabalho, exaustão física e mental (CHUNG, 2020).

Em um estudo realizado por Wiersinga (2020), apontando os principais grupos de risco desta comorbidade, onde é enfatizado a classe trabalhista dos profissionais de saúde, sendo apontada a exposição ao vírus como um dos principais fatores de risco, sendo considerado também outros grupos como Idosos acima de 60 anos de idade; População indígena, Portadores de asma, tuberculose, cardiopatias, nefropatias, hepatopatias, doença hematológica como a anemia falciforme, distúrbios metabólicos como a diabetes mellitus, transtornos neurológico, imunossuprimidos e obesos com IMC > 40 .

Os profissionais de saúde enfrentam variados problemas no que diz respeito a sua saúde, pois estes expõem-se ao contato prologado com seus pacientes infectados pela Covid, sendo comum acontecer descuidos ocasionando alta exposição ao vírus, muitas vezes ocasionados pela exaustão ou até mesmo estresse devido a sobrecarga de trabalho entre outros motivos, sendo necessário haver a existência de protocolos que visem minimizar os riscos (TEIXEIRA et al., 2020).

Para Gallasch, Cunha, Pereira e Silva-Junior (2020), a implantação de ações voltadas para proteção dos trabalhadores da saúde, no que diz respeito ao contato prolongado com paciente acometidos por covid-19, devendo ser de grande segurança, mas a grande fragilidade é o fornecimento dos EPI's, assim como também o uso manejo desses insumos, onde os autores evidenciaram que para alcançar uma resposta adequada, profissionais da área de saúde precisam ser previamente preparados, equipados e informados quanto às medidas de controle da propagação da infeção.

Os fatores de risco relacionados à infecção pelo SARS-CoV-2 mais citados foram: escassez de Equipamentos de Proteção Individual, sobrecarga de trabalho, uso inadequado ou não uso de EPI e higiene inadequada das mãos, contato próximo com pacientes e/ou colegas de trabalho potencialmente contaminados, procedimento com risco de geração de aerossol, diagnóstico de COVID-19 tardio e renovação de ar insuficiente em ambiente de pressão negativa.

O estudo de Vedovato e seus colaboradores (2021) que objetivou averiguar as condições de trabalhos durante a pandemia evidenciou que estas são 
inadequadas pela inexistencia ou precariedade dos EPI's, profissionais exercendo suas funções trabalhistas mesmo encontrando-se infectados, o medo de contrar a infecção do covid, dificuldades no acesso aos testes de COVID-19 e para afastamento do trabalho para tratamento.

A Sociedade Brasileira de Infectologia preconiza a utilização dos EPI's com o objetivo de proteção a gotículas ao contato de pacientes sintomáticos respiratórios, devido a covid, onde existe uma ressalva relacionada a má manipulação desses equipamentos, sendo ressaltado o uso de insumos insuficientes, como acontece no uso de apenas as máscaras, pois essas não inibem as demais vias de contaminação como por exemplo a ocular, onde a falta desses matérias chega a $38,2 \%$ em ambientes hospitalares no Brasil (CARVALHO et al., 2020).

Os profissionais de saúde que atuam na linha de frente evidenciaram ter um maior risco de apresentar sintomas psicológicos e transtornos mentais. Esses trabalhadores são encarregados a prestar essa assistência direta no cuidado a pacientes com Covid-19 e encaram fatores de risco psicossociais característico como cansaço físico e mental devido ao uso ininterrupto de EPIs, a angústia pela falta de tratamento para a doença e a falta de amparo (BUSELLI et al., 2021).

Além desses fatores citados, vários outros contribuíram para o aumento de transtornos mentais. O medo de se contaminar, o aumento de óbitos de pacientes portadores de Covid-19, a carência de insumos e medicamentos, a falta de EPIs, cansaço físico e o aumento da carga horária de trabalho (KHAMIS et al., 2021). Em um estudo no qual avaliou a saúde mental dos profissionais da saúde durante a pandemia em um determinado hospital houve prevalência de ansiedade $(48,9 \%)$ e de depressão (25\%). (DAL'BOSCO, 2020).

Em uma pesquisa realizada por estudo Zhang e seus colaboradores (2020), observou uma prevalência onde $73,4 \%$ dos profissionais de saúde estudados, num total de 1.563 trabalhadores apresentavam estresse, 50,7\% depressão e insônia em $36,1 \%$.

Para Helioterio e seus colaboradores (2020) em sua pesquisa realizada em hospitais no Rio de Janeiro na rede pública observou-se uma taxa elevada de profissionais de saúde infectados pelo covid-19 (25\%), onde ao ser comparado com os estudos de Azevedo (2020), Koh (2020) e Filippo e seus colaboradores (2020) 
nos quais foram averiguados uma taxa diferente da brasileira onde na China (4\%) e na Itália (15\%).

Os meios de transmissão de SARS-CoV-2 são diversos: contato entre pessoas infectadas através das gotículas salivares que se espalham em meio ao ar pela fala, tosse, espirra, podendo atingir a boca, nariz e olhos, dispersão do vírus no ar que são gerados em alguns procedimentos realizados em ambiente hospitalar, com isso existe um aumento das chances do profissional de saúde contaminar-se devido o contato prologado com o paciente e uma maior exposição ao vírus, além disso existe também o contato com objetos infectados como estetoscópio, termômetro e outros materiais que estejam sendo usados em pessoas infectadas. (OPAS, 2020). Dessa forma é possível entender que o trabalhador da saúde, está inserido em um ambiente minado por diversos fatores de risco predisponentes a infecção do vírus, podendo gerar desequilíbrio biopsicossocial.

\section{CONCLUSÃO}

Concluindo, a saúde dos profissionais foi afetada pelo fato dos mesmos possuírem uma maior chance de adquirir a patologia, assim como também suas complicações, pois este público encontra-se mais exposto devido uma maior exposição ao vírus, a falta de EPl's entre outros fatores apontados neste estudo, além de comprometer a saúde física pode também afetar a psicológica.

Destacam-se como recomendações: revisão de fluxos de atendimento e definição de características e condições para cada etapa de atendimento; estabelecimento da Covid-19 como doença relacionada ao trabalho para os grupos expostos; acompanhamento de suporte psicológico, melhor distribuição de EPI's, assim como também reciclagem sobre seu uso correto, registro efetivo da 'ocupação' nos sistemas de informação. 


\section{REFERÊNCIAS BIBLIOGRÁFICAS}

BRASIL, Ministério da Saúde. Coronavírus-Sobre a doença/ Ministério da Saúde- Brasília-DF, Ministério da Saúde, 2020. Disponível em: https://coronavirus.saude.gov.br/sobre-adoenca\#interna Acesso em 28 de maio de 2021.

BURRER, S., PERIO, M., HUGHES, M., KUHAR, D., LUCKHAUPT, S., \& MCDANIEL, C. (2020). Características do Pessoal de Saúde com COVID-19. MMWR Morb Mortal Wkly Rep., 69(15), 477-481.

BUSELLI, R., Corsi, M., BALdANZI, S., CHIUMiENTO, M., Del Lupo, E., DELL'OSTE, V., BERTELLONI, C. A., MASSIMETTI, G., DELL'OSSO, L., CRISTAUDO, A., \& CARMASSI, C. (2020). Professional Quality of Life and Mental Health Outcomes among Health Care Workers Exposed to Sars-Cov-2 (Covid-19). International journal of environmental research and public health, 17(17), 6180. https://doi.org/10.3390/ijerph17176180.

CA, Massimetti G, Dell\&\#39; Osso L, CRISTAUDO A, CARMASSI C. Professional Quality of Lifeand Mental Health Outcomes among Health Care Workers Exposed to Sars-Cov-2(Covid-19). Int J Environ Res Public Health. 2020 Aug 26;17(17):6180. doi:10.3390/ijerph17176180. PMID: 32858810; PMCID: PMC7504107.

CARVALHO, R., AUGUSTO, G., SCHOEN, I., Oliveira, Y., \& Zibordi, V. (2020). Utilização de equipamentos de proteção individual em época de COVID-19. Glob Acad Nurs.

CHUNG JPY, YEUNG WS. Staff Mental Health Self-Assessment During the COVID-19 Outbreak. East Asian Arch Psychiatry. 2020. Disponível em: <https://doi.org/10.1590/00472085000000316>.

GALLASCH, C., CUNHA, M., PEREIRA, L., \& SILVA-Junior, J. (2020). Prevenção relacionada à exposição ocupacional do profissional de saúde no cenário de COVID-19. Rev enferm UERJ.

HELIOTERIO, M., LOPES, F., SOUSA, C., SOUZA, F., PINHO, P., SOUSA, F., \& ARAÚJO, T. (2020). Covid-19: Por que a proteção de trabalhadores e trabalhadoras da saúde é prioritária no combate à pandemia? Trab. Educ. Saúde.

KHAMIS F., AI MAHYIJARI N., AI LAWATI F., BADAHDAH AM. A saúde mental de médicas e enfermeiras em Omã durante a pandemia de COVID-19. Oman Med J. 2020; 35 (6): e203. Publicado em 30 de novembro de 2020. doi: 10.5001 / omj.2020.103.

OPAS. (2020). Transmissão do SARS-CoV-2: implicações para as precauções de prevenção de infecção.

PRADO A. D.; PEIXOTO B. C.; da SILVA A. M. B.; SCALIA L. A. M. A saúde mental dos profissionais de saúde frente à pandemia do COVID-19: uma revisão integrativa. Revista Eletrônica Acervo Saúde, n. 46, p. e4128, 26 jun. 2020. Disponível em: https://doi.org/10.25248/reas.e4128.2020.

RIBEIRO, A., OLIVEIRA, G., SILVA, L., \& SOUZA, E. (2020). Saúde e segurança de profissionais de saúde no atendimento a pacientes no contexto da pandemia de Covid-19: revisão de literatura. Rev. bras. saúde ocup., 45(25).

TEIXEIRA, C., SOARES, C., SOUZA, E., LISBOA, E., PINTO, I., ANDRADE, L., \& ESPIRIDIÃO, M. (2020). A saúde dos profissionais de saúde no enfrentamento da pandemia de Covid-19. Ciênc. saúde coletiva . 
VEDOVATO, T., ANDRADE, C., SANTOS, D., BITENCOURT, S., ALMEIDA, L., \& SAMPAIO, J. (2021). Trabalhadores(as) da saúde e a COVID-19: condições de trabalho à deriva? Rev Bras Saude Ocup.

WIERSINGA, W., RHODES, A., CHENG, A., PAVÃO, S., \& PRESCOTT , H. (2020). Fisiopatologia, Transmissão, Diagnóstico e Tratamento da Doença Coronavírus 2019 (COVID19): Revisão. Jama, 324(8), 782-793.

ZHANG, C., YANG, L., LIU, S., MA, S., WANG, Y., CAI, Z., ... ZHANG, B. (2020a). Survey of insomnia and related social psychological factors among medical staff involved in the 2019 novel coronavirus disease outbreak. Frontiers in Psychiatry, 11(306), 1-9. 